to the point of restlessness, or were flitting in play. Exchanges between mother and child tended to be reduced and to lack warmth. The mothers tended to express dissatisfaction with the child, although there was also some concern over the difficulties in feeding and his shortness. This was often attributed to inheritance, a short parent or grandparent being mentioned. The decision to seek advice had usually been made when comparison with other children had shown up the degree to which growth had lagged.

Advice given-At the first attendance we sought information about appetite, feeding habits, and diet. Once the dietary assessment had been made we discussed these matters at each visit in as relaxed a way as we could. We encouraged the mothers to give the child as wide a choice of food as possible at each meal in cafeteria or smörgassbord style. Occasionally we gave advice about supplementing the diet, recommending special highenergy foods when appropriate. At one stage we embarked on a placebo-controlled trial of cyproheptadine $4 \mathrm{mg}$ twice daily for six months as an appetite stimulant, ${ }^{13}$ but the results were poor and the trial was abandoned. We think that the increase in calorie content of the diets recorded at the second assessment was due, in part at least, to a change in the mothers' and children's attitudes as a result of the discussions at the clinic.

\section{Conclusions}

The hypothesis that retarded growth in otherwise healthy children is due to a long-continued deficiency in calorie intake gains some support from our findings within the limits set by the scope and methods of the study. The following recommendations are based on our experience over several years.

(1) The monitoring of development during childhood should include regular measurement of height and its recording on a chart.

(2) If growth lags, inquiries should be made into the child's feeding habits. Details of the diet should be obtained and recorded, even when an accurate assessment of the calorie content cannot be made.

(3) Interest in these matters should not divert attention from the indications and investigation of organic disease, and the physician should be alert for other causes. An endless repetition of investigations for a physical cause of stunted growth, however, is likely to increase anxiety and be self-defeating.

(4) Relaxed, repeated discussion of any feeding problems or deficiency in the diet may be expected to bring about an improvement in many cases, though it may be many months before this shows. A sustained increase in the calorie content of the diet should resul ${ }^{+}$in more rapid growth.

(5) Some catching ur oft $\leq n$ takes place. The pubertal growth spurt tends to occur, although it may be delayed. A spurt of this kind may be associated with a change in feeding habits and improvement of the diet. Among the many facets of general management this encouraging expectation should be discussed with the parents and child.

\section{References}

${ }^{1}$ Apley, J, et al, Proceedings of the Royal Society of Medicine, 1971, 64, 135. 2 Shields, J, Monozygotic Twins Brought Up Apart and Brought Up Together. London, Oxford University Press, 1962.

3 Topp, S G, et al, British fournal of Social and Preventive Medicine, 1970, 24, 154.

${ }^{4}$ British Medical fournal, 1976, 1, 359.

5 Harris, F, Medicine, 1974, 27, 1600.

${ }_{6}$ Lacey, K A, and Parkin, J M, Lancet, 1974, 1, 42.

${ }^{7}$ Parkin, J M, Archives of Disease in Childhood, 1974, 49, 904.

${ }^{8}$ MacCarthy, D, and Booth, E M, Fournal of Psychosomatic Research, 1970, 14, 259.

${ }^{9}$ Lacey, K A, and Parkin, J M, Archives of Disease in Childhood, 1974, 49, 417.

${ }^{10}$ Tanner, J M, et al, Assessment of Skeletal Maturity and Prediction of Adult Height (TW2 Method). London, Academic Press, 1975.

11 Dean, R F A, Modern Problems in Pediatrics, 1960, 5, 111.

12 Waterlow, J C, Lancet, 1973, 2, 87.

13 Penfold, J L, Medical fournal of Australia, 1971, 1, 307.

(Accepted 13 December 1977)

\title{
Human leucocyte antigens and mixed lymphocyte reaction in severe pre-eclampsia
}

\author{
D M JENKINS, JILLIAN A NEED, J S SCOTT, HELEN MORRIS, M PEPPER
}

British Medical fournal, 1978, 1, 542-544

\section{Summary and conclusions}

Human leucocyte antigens (HLA) and mixed lymphocyte reactions (MLR) were studied in 38 women with severe pre-eclampsia and their husbands. Thirty-nine women with normal pregnancies and their husbands served as

\footnotetext{
Department of Obstetrics and Gynaecology, Leeds Maternity Hospital, University of Leeds, Leeds LS2 9NG

D M JENKINS, MD, MRCOG, senior lecturer

JILLIAN A NEED, MRCOG, research fellow (present address: Department of Obstetrics and Gynaecology, Flinders University of South Australia, Bedford Park, South Australia 5042)

J S SCOTT, FRCSED, FRCOG, professor

HELEN MORRIS, BSC, research assistant
}

Regional Blood Transfusion Service, Bridle Path, Seacroft, Leeds M PEPPER, FIMLT, senior technician controls. Thirty-three of the control women were matched for age and parity with members of the study group. Infants were studied when possible. HLA compatibility was increased in the pre-eclamptic group compared with matched controls and with theoretical estimates for possible matings.

The one-way MLR at delivery showed diminished response of maternal to paternal and cord cells in preeclamptic women. This reduced maternal reactivity in women with pre-eclampsia may have a role in the illness, and paternal/maternal histocompatibility may be a feature of the severe form.

\section{Introduction}

The fetus, which inherits half of its histocompatibility antigens from the father, is an allograft on the maternal host. Its survival remains an immunological paradox, and the cause of the major disease in pregnancy, pre-eclampsia, is still unknown. As immunogenetic factors may be relevant ${ }^{1}$ we undertook a con- 
trolled study of the distribution of human leucocyte antigens (HLA) and mixed lymphocyte reactions (MLR) in severe pre-eclampsia.

\section{Patients and methods}

Patients were chosen by screening 45000 pregnancies occurring over three years in a population of about one million in the Leeds/ Bradford conurbation. Thirty-eight women with severe pre-eclampsia formed the study group. The diagnostic criteria for pre-eclampsia were hypertension of at least $160 / 110 \mathrm{~mm} \mathrm{Hg}$ on two or more occasions, developing in late pregnancy and settling after delivery, and proteinuria of at least $3 \mathrm{~g} / \mathrm{l}$. Thirty-nine women with normal pregnancies served as controls, 33 of them being matched for age and parity with members of the study group. Babies were included when possible, but perinatal mortality was high and fresh cord blood was not always available for study. Husbands were therefore tested, as the fetal HLA haplotype, which is unlikely to be shared by the mother, is of paternal origin.

HLA types of both groups of women and their husbands were studied. Twenty HLA antigens were typed using at least two antisera for each; ABO typing was also performed. Data from the control group and estimates for matching of alleles in hypothetical random matings in the general population were used for comparison. The HLA types of the husbands of the pre-eclamptic women were used as internal controls for comparisons with the HLA types of each of the other pre-eclamptic women.

Mother-father and mother-child MLR studies were performed at delivery in 25 of the pregnancies complicated by severe pre-eclampsia and in 33 controls. A whole-blood MLR method was used. ${ }^{2}$ Allogeneic cultures were done after the mothers' serum had been removed by washing the cells three times in culture medium before dilution. The stimulating paternal or cord lymphocytes were treated with mitomycin. Maternal lymphocytes were also cultured with mitomycintreated maternal lymphocytes (isogeneic mixture) to provide a "stimulation index" based on the allogeneic: isogeneic response ratio for comparison.

\section{Results}

HLA-antigen distribution-The distribution of HLA antigens in patients with severe pre-eclampsia or their husbands compared with controls was not significantly different after statistical correction for the number of typed antigens.

HLA-antigen matching-The incidence of matching of HLA antigens in pre-eclamptic women and their husbands was greater than in the control pairs. The difference was not significant for any single antigen. Eleven out of 13 antigens were more commonly matched in pre-eclamptic women and their husbands than in the 33 control pairs. Eighteen out of 20 antigens (see fig) were found to be more often matched in pre-eclamptic pairs than expected from random matings $\left(\chi^{2}=5.83 ; P<0.05\right)$. If matching of antigens between pre-eclamptic women and their husbands was not related to the risk of developing the disease the points on the display ${ }^{3}$ for each antigen would be scattered randomly about unity. HLA matching was found to be more common in actual married pairs than hypothetical pairs. The

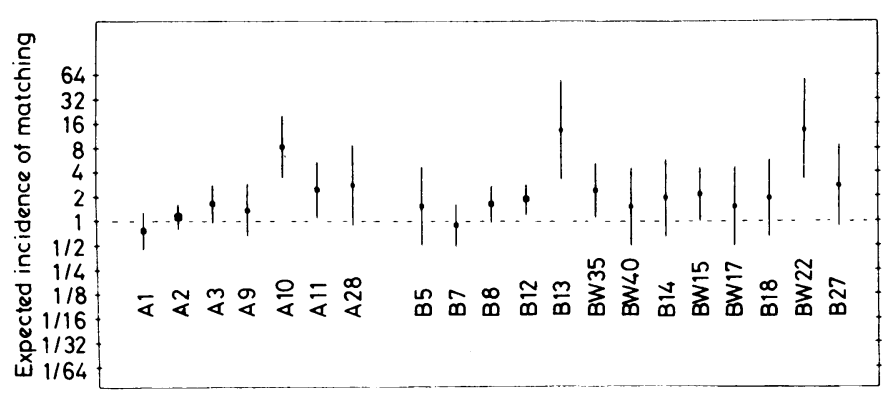

Matching of HLA antigens in pre-eclamptic pairs compared with estimates from general population data. Squares on display relate in size to frequency of matchings $( \pm S D)$. Marks on vertical axis represent doubling or halving in incidence of matching from that expected. Eighteen of the 20 antigens were more commonly matched in pre-eclamptic pairs than controls. (See Edwards. $^{3}$ ) mean number of matched antigens was $1 \cdot 0 \pm S D 0.84$ for actual married pairs and $0.7 \pm 0.83$ for the hypothetical pairs. Similar estimates within the control group of women with normal pregnancies showed the mean number of shared antigens to be $0.64 \pm 0.53$ in actual married pairs and $0 \cdot 7 \pm 0.82$ for all other possible pairings within that group.

Mean $( \pm S D)$ values for one-way mixed lymphocyte reactions $(M L R)$ performed at delivery in 25 pre-eclamptic women and 33 normal controls and their husbands and babies*

\begin{tabular}{|c|c|c|c|}
\hline MLR study & $\begin{array}{l}\text { Pre-eclamptic } \\
\text { patients }\end{array}$ & Controls & $\mathbf{P}$ value $\dagger$ \\
\hline $\begin{array}{l}\text { Mother-father } \\
\text { Mother-child }\end{array}$ & $\begin{array}{l}1 \cdot 35 \pm 1 \cdot 19 \\
(n=25) \\
0 \cdot 59 \pm 1 \cdot 26 \\
(n=11)\end{array}$ & $\begin{array}{l}2 \cdot 62+1 \cdot 76 \\
(n=33) \\
1 \cdot 38+1 \cdot 50 \\
(n=30)\end{array}$ & $\begin{array}{c}<0.01 \\
\text { NS }\end{array}$ \\
\hline
\end{tabular}

*Studies on fresh cord blood were sometimes not possible due to perinatal deaths. +Two-sample rank sum test. NS - Not significant.

Infants - In 19 pairs of pre-eclamptic mothers and babies the mean number of matched antigens for each pair was 1.8 , and in 17 control pairs 1.4 ; the difference was not significant.

$A B O$ data-The presence or absence of maternal $\mathrm{AB}$ isoantibody to baby's $A B O$ group was not associated with a changed incidence of HLA-antigen matching in pre-eclamptic women and their husbands or babies.

$M L R$ studies-One-way MLRs of maternal to paternal and maternal to cord cells performed at delivery showed a significantly diminished reactivity $(P<0.01)$ between maternal and paternal cells in preeclamptic patients compared with controls (see table).

\section{Discussion}

Our failure to show any relation between a particular HLA antigen and pre-eclampsia agrees with the results of a similar study of 46 women with pre-eclampsia or eclampsia. ${ }^{4}$ The fetus is a homograft, however, and the relation between the different HLA types may be relevant in pre-eclampsia. Previous consideration of immunogenetic interaction between conceptus and mother in pre-eclampsia concentrated on the possibility of a hyperimmunity response by mother or fetus to some antigen in the other. ${ }^{1}$ Such a response has never been convincingly shown. Furthermore, we found production of maternal HLA antibody to be absent in the pre-eclamptic mothers. ${ }^{5}$ Such a maternal response would be inconsistent with the epidemiological evidence of a higher incidence of pre-eclampsia in first pregnancies.

Increased matching of HLA antigens in the parental pairs in severe pre-eclampsia was associated with reduced maternal immunological reactivity as assessed by the MLR. The one-way MLR measures the active immune response of the mother's lymphocytes to stimulation by the inactivated (paternal or cord) cells. Stimulation is controlled by MLR determinants which are coded for by a locus closely linked to but independent of the loci coding for the serologically defined HLA antigens. A recent report $^{6}$ also provided some evidence of reduced lymphocyte reactivity in pre-eclamptic pregnancies. The MLR findings alone would be of limited importance, but when considered with the paired HLA data they seem to show the same type of immunological relation with a lower degree of maternal reactivity in the pre-eclamptic patients. The data on the number of matched antigens in each couple also suggest that relative histocompatibility is a feature of severe pre-eclampsia. Unlike many other abnormalities associated with pre-eclampsia these antigen findings could not have been a consequence of the disease process.

There has been much confusing evidence on $\mathrm{ABO}$ blood grouping and pre-eclampsia. ${ }^{1}$ This small series does nothing to clarify this, but study of HLA and ABO types in the same patients may show that the two systems are interrelated, as is the case with artificial homografts.?

Reduced maternal incompatibility and immune response to 
the conceptus antigens may be a factor in the aetiology of preeclampsia. This theory does not seem to have been considered before and certainly was not in our minds at the outset. It may explain why the disease is more common in first pregnancies, which usually represent the first exposure to conceptus antigens.

This study was supported by Medical Research Council grant No $974 / 52 \mathrm{C}$. We thank our obstetric colleagues for allowing us to study their patients; Professors J H Edwards and T P Whitehead, of the Medical School, Birmingham, for the use of computing facilities and equipment; Mrs Elaine Hunter for technical help; and Dr S M Rajah and technical staff of the Regional Blood Transfusion Service, Bridle Path, Leeds, for the tissue typing.

Details of the ABO and HLA typing of the patients in the study may be obtained from Professor J S Scott, Department of Obstetrics and Gynaecology, 17 Springfield Mount, Leeds LS2 9NG, to whom requests for reprints should also be addressed.

\section{References}

1 Scott, J S, and Jenkins, D M, Fournal of Medical Genetics, 1976, 13, 200.

2 Need, J A, British Medical fournal, 1975, 1, 548.

3 Edwards, J H, fournal of Immunogenetics, 1974, 1, 249.

${ }_{4}$ Scott, J R, and Beer, A E, Fournal of the American Medical Association, 1976, 235, 402.

${ }^{5}$ Jenkins, D M, Need, J, and Rajah, S M, Clinical and Experimental Immunology, 1977, 27, 485.

${ }^{6}$ Gille, J, Williams, J H, and Hoffman, C P, European fournal of Obstetrics, Gynecology and Reproductive Biology, 1977, 7, 227.

7 Opelz, G, and Terasaki, P I, Lancet, 1977, 1, 220.

\title{
Immunological detection of residual leukaemic disease in the bone marrow of children with acute lymphoblastic leukaemia
}

\author{
SHANT KUMAR, TREVOR F CARR, IAN M HANN, PATRICIA H MORRIS JONES, \\ DAVID I K EVANS
}

British Medical fournal, 1978, 1, 544-546

\section{Summary and conclusions}

Peripheral blood lymphocytes incubated with tumour cells or extracts may undergo blastogenesis. This is the basis of a technique studied in children with acute lymphoblastic leukaemia (ALL) in childhood in an attempt to predict relapse. Samples of peripheral blood and bone marrow from 82 children with varying degrees of ALL were analysed. Cultures were prepared by incubating a lymphocyte suspension with an autologous bone-marrow suspension. Final ratios of lymphocytes to bone-marrow cells ( $L: B M)$ were $1: 1$ and $2: 1$. Control wells received bone-marrow or lymphocyte suspension only. Cultures were incubated for 72,96 , and 120 hours. All were pulse-labelled with ${ }^{3} \mathrm{H}-\mathrm{TdR}$ and radioactivity was measured by scintillation counting. Results were expressed as the stimulation index, calculated by dividing the mean counts per minute ( $\mathrm{cpm}$ ) of wells containing both lymphocytes and bone-marrow cells by the sum of the mean cpm for control wells. If the stimulation index exceeded 1 at 72, 96, or 120 hours at either $L: B M$ ratio a positive response was recorded.

Seventy-six children were in clinical remission at the time of testing (group A) and six were in clinical relapse (group B). In group A 24 patients showed stimulation

Christie Hospital and Holt Radium Institute, Withington, Manchester M20 9BX

SHANT KUMAR, MVETSC, PHD, head, paediatric oncology laboratory TREVOR F CARR, HNC, technician

The Royal Manchester Children's Hospital, Pendlebury, Manchester M27 1HA

IAN M HANN, MB, MRCP, research senior registrar, department of paediatric oncology and haematology

PATRICIA H MORRIS JONES, MB, MRCP, senior lecturer, department of child health, paediatric oncology

DAVID I K EVANS, MRCP, DCH, consultant haematologist and relapsed later at a mean time of 3.8 months (21 with marrow disease, two with testicular infiltration, and one with lung infiltration). Sixteen patients showed stimulation and had up to $4 \%$ blasts in their bone marrow but remained in remission. Nineteen other patients showed a positive response and several factors may have contributed to this: two underwent a "rebound" lymphocytosis after stopping treatment, nine had current or intercurrent infections, two had persistent unexplained bone-marrow lymphocytosis, but six had no causative symptoms and thus their responses were "true falsepositives." Seventeen patients from group A showed no response and remained in remission for a mean of 22.9 months after testing. None of the six children in group $B$ responded, and at testing had $17-85 \%$ blasts in their bone marrow.

During the study no patient relapsed who had not shown a positive response. The technique merits further study as a guide to the presence of leukaemic cells.

\section{Introduction}

The reduction of lymphoblasts to $5 \%$ or less in bone-marrow smears is one of the commonly used criteria ${ }^{1}$ of clinical remission in acute lymphoblastic leukaemia (ALL). Blasts are recognisable mainly morphologically and rarely cytochemically, ${ }^{2}$ and the limitations of these methods are well known. A more sensitive diagnostic technique than the staining methods provide is needed to detect such a small number of leukaemic blasts. Such a test would improve the clinical management of patients, since leukaemic cells detected in a patient otherwise considered to be in remission would indicate the need for additional or intensive chemotherapy, or both, which should increase the number of long-term survivors. The test could also be used in deciding whether to stop or continue chemotherapy in a particular case.

Peripheral-blood lymphocytes incubated with intact tumour cells or tumour-cell extracts may undergo blastogenesis, ${ }^{3}$ which is detected morphologically or by the increase of DNA synthesis. ${ }^{4}$ Autologous non-neoplastic cells have little or no such stimulatory effect. The difference has been interpreted as a 